Available Online at www.ijcsmc.com

\title{
International Journal of Computer Science and Mobile Computing
}

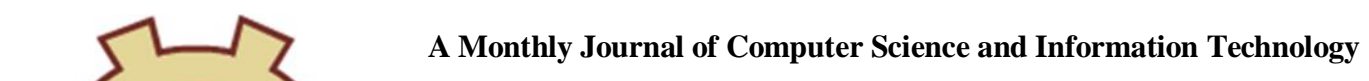

ISSN 2320-088X

IJCSMC, Vol. 10, Issue. 6, June 2021, pg.83 - 89

\section{DESIGN \& IMPLEMENTATION OF POLYPHASE FILTER TO DENOISE OFDM SIGNALS}

\author{
*GOUSIA NABI DAR; **Er. RAJAT JOSHI \\ *Department Of ECE, Adesh Institute Of Technology, Chandigarh \\ **DIRECTOR Adesh Institute Of Technology, Chandigarh
}

DOI: 10.47760/ijesmc.2021.v10i06.009

\begin{abstract}
An amazingly effective digital transit system that carries multiple carriers that meet in conjunction with each other over a period of time is known as the Orthogonal Frequency Division Multiplexing (OFDM) system. Among the traditional symbols, there is a need to include a group of guards. However, in OFDM systems this is not required. Although there is an overlap of side bands from each carrier, no interference is involved within the signals found here as they are orthogonal in relation to each other. This research work is based on a wireless channel to reduce the rate of error using space-time trellis codes. In this research project, the minimum error rate is reduced over wireless channels using space time codes and poly-phase filters. The proposed modular simulation is performed at MATLAB and the results show that the minimum bit error rate decreases in the network.
\end{abstract}

KEYWORDS: Signal Denoising, OFDM, Polyphase Filter

\section{Introduction}

Wireless communication can be defined as the process by which data is transmitted to two places that are not physically connected to each other. By providing connectivity to wireless technology, electric waves are used. On the basis of the various applications in which communication is used, these waves travel over distances. Several scheduled, mobile and portable applications include that form of communication. There are two-way radios, digital assistants (PDAs) and various other devices included here. Other features such as keyboards, radio receivers, headsets etc. are included in wireless radio applications and [1]. By providing communication, light, sound, magnetic or electric fields are integrated into wireless communication technology. However, compared with the spirits mentioned above, these means of communication are less commonly used. Orthogonal frequency-division 
multiplexing (OFDM): OFDM is a technology in which digital data is integrated into multiple frequency carriers. There are wireless and wireless connections available within the digital wideband connection. Therefore, OFDM programs have also begun to use these two approaches. Multiple-input and multiple-output (MIMO) is defined as a technology in which the transmitter and receiver ends up having multiple horns [2]. In the programs available from the end of the receiver, there are several copies provided for the same type of information signal. From more than two or more real communication channels, transfers of these copies can be made. Basic function provided within variations in duplication or lack of data available on networks. In a variety of ways, decisions are made by the recipient and the sender also does not know about them. In addition to signal transmission, several distribution methods are available [3]. The occurrence of a process that withers within a wireless channel can lead to signal exposure to certain broadcast media. There are variations in blurring in time, location or frequency of the radio used. Processing here is done randomly. The disappearing channel is known to be the one where there is a connection and the end is included. Blurring can be caused by wireless systems due to multipath proliferation or due to irritability [4]. A signal is a function of independent variable, for example, time, distance, position, temperature and pressure. The signal transmits data, and the purpose of the signal processing is to extract important information transmitted by the signal. Signal analysis is related to the statistical representation of the signal and the algorithmic functionality used to extract the available data. The rapid advances in science and engineering are the result of significant advances in digital computer technology and integrated circuit design [5]. Digital computers and compatible digital hardware for the past three decades were very large and expensive and, as a result, their use was limited to non-real (disconnected) scientific statistics and business applications. Electrical appliances are used extensively as part of almost all sectors. Analog to Digital Converters (ADC) and Digital to Analog Converters (DAC) are the key elements in any DSP variation in any field. These two flexible associations are essential for converting real-world signals to consider digital electronic devices to detect any analog signal and process it. Digital filters are an integral part of DSP. Truth be told, their unusual performance is one of the main reasons why DSP has emerged so popular. Filters have two uses: signal separation and signal replacement. There are five fundamental filter types (bandpass, notch, low-pass, high-pass, and all-pass). The number of possible bandpass response characteristics is infinite, yet they all share a similar fundamental form [6]. The curve present in the graphs of bandpass may be called an "ideal" bandpass response, with totally constant gain inside the passband, zero gain outside the passband, and an abrupt boundary between the two. A filter with effectively the opposite function of the bandpass is the band-reject or notch filter. Notch filters are utilized to remove an unwanted frequency from a signal, while affecting all other frequencies a little less. A third filter type is the low-pass. A low-pass filter passes low frequency signals, and rejects signals at frequencies over the filter's cutoff frequency. The opposite of the low-pass is the high-pass filter, which rejects signals below its cutoff frequency [7]. A high-pass filter can be made by rearranging the components. High-pass filters are utilized as a part of applications requiring the rejection of low-frequency signals. A FIR filter requires more computation time on the DSP and more memory. The DSP chip in this way needs to be all the more powerful. miniDSP products that support FIR filtering include the OpenDRC and the miniSHARC kit. A FIR filter is designed by finding the coefficients and filter order that meet certain specifications, which can be in the time-domain (e.g. a matched filter) and additionally the frequency domain

\section{Literature Review}

Hen-Geul Yeh, et.al (2016) introduced that in OFDM systems, coding availability and diversity are provided and ensured that bandwidth efficiency is not reduced through the use of Space-time trellis code (STTC) techniques. To improve BER within selected mobile blurring channels, three OFTCM-based STTC systems are presented in this paper [8]. Compared to existing STTC-WH-ST-OFDM and STTC-ST-OFDM programs, the proposed STTC-WHSTCC-OFDM system proves to be better in terms of simulation results. The compatibility of all three systems is good with regard to OFDM systems and is easy to implement. In many OFDM programs, these algorithms serve as blocks of baseband construction. 
Neethu V, et.al (2017) proposed a novel process to improve the overall functionality of OFDM systems [9]. The trellis coded orbital angular momentum- quadrature amplitude modulation (OAM-QAM) and advanced time-varying frequency (eTFM) methods are combined to form a novel approach. Data mapping with trellis codes for OAMQAM star locations is also done using a viterbi decoder, this data is visible. By ensuring that bandwidth is not expanded, the encoding benefit is enhanced here. Compared with traditional methods, the BER value is reduced due to the increase in Euclidean distance. Therefore, there is an improvement in the effectiveness of the results obtained through the proposed process.

Hushou Chen, et.al (2018) proposed a novel algorithm to reduce the maximum power rating (PAPR) within OFDM markers [10]. Within a small trellis of block codes, partial transfer sequence (PTS) is performed here. To select a OFDM signal signal with a small PAPR, direct code is used that incorporates a beautiful trellis. With error correction, the additional information is transferred. As the simulations performed and the results obtained it is evident that the complexity is reduced and the reduction of PAPR through the proposed procedure.

Samet Yıldız, et.al (2016) suggested an advanced method of obtaining high probability to separate individual signals sent to the appropriate receiver [11]. Here, on the transmitter, a multiplexer is used and on the receiver, a demultiplexer is used. Improved frame rate (FER) error rate performance is achieved using STTC-OFDM as the test performed. In order to compile the system in such a way that the proposed and existing methods can be compared, the Doppler effect is included here. Improvements in simulation results indicate that the proposed method is better compared to other existing methods.

Ryota Yoshizawa et.al (2016) suggested a novel star formation where at the end of the receiver, the controlling pieces and the details of the information could be completely separated [12]. The trellis-based limit made by the memory bank is used to control the fragments. In addition, with the proposed system, the composition of the novel star is appropriate. In the OFDM coded system, a simple process that includes a soft-in soft-out (SISO) decoder is performed here. The effectiveness of the proposed system is evaluated according to specific operating principles. Moreover, the apparent advantage of this method in the high-spectral system is reflected in the comparisons made between the proposed methods and the few existing ones.

Funmilayo B. Offiong, et.al (2016) suggested a novel driver's assist in this paper in which the reduction of the average power in which they used multiplexing frequency multiplexing systems in this paper reduced. DCO-OFDM and ACO-OFDM are the two programs studied in this paper, these are the domain symbols of time. They have used the Gaussian distribution and the Gaussian component in their programs to achieve better performance [13]. Due to the higher value of $\mathrm{P}$ and due to the minimization of PAPR, there is huge complexity. The better results have been provided by this method by which optical energy per bit to noise power spectral thickness ratio has been minimized. They performed various experiments and comparisons on the basis of various parameters and concluded that proposed method has better performance as compared to other methods

\section{Research Methodology}

To mitigate ICI, the 2x1 STCC-OFDM systems are developed which mainly stimulate the two-way transmission mechanism of the CC-OFDM scheme. Most existing OFDM systems are backwards compatible with STCC-OFDM systems. By selecting time division multiplexing (TDM), frequency division multiplexing (FDM) or code division multiplexing (CDM), the multiplexing circuit (MUX) is applied to the transmitter and multiplexing circuit (DEMUX) at the receiver. Through these steps, the STCC-OFDM systems are designed. 


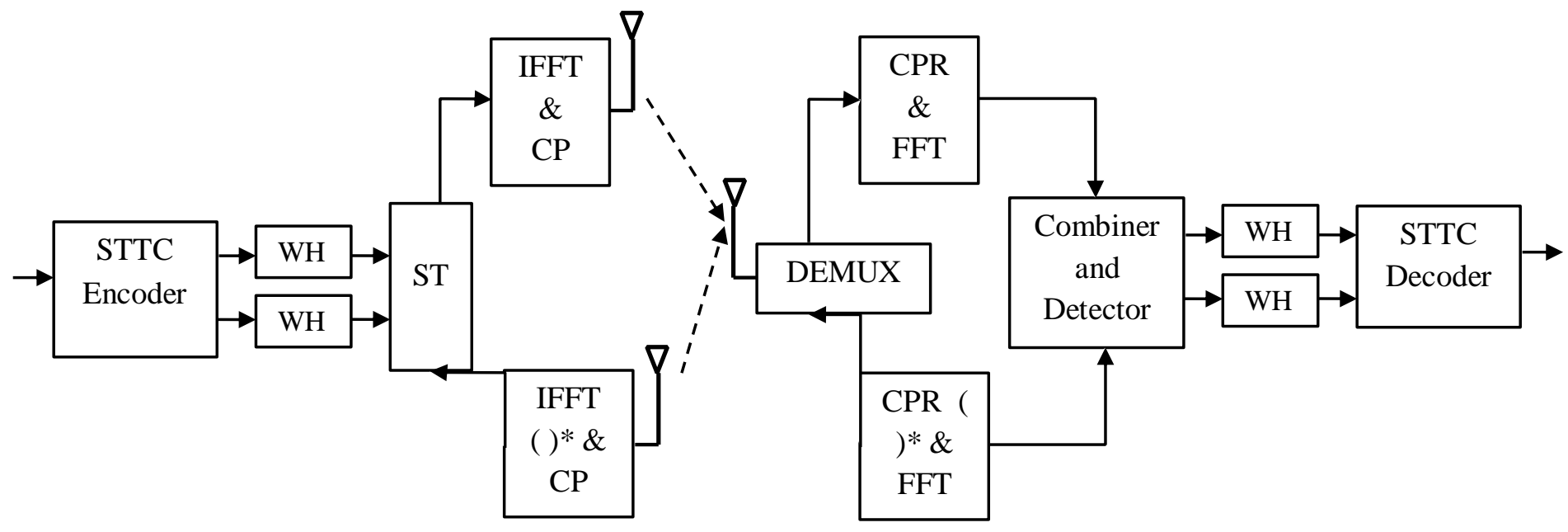

Figure 1: The architecture of a 2x1 STTC-WH-STCC-OFDM System with WHT pre-coders and CC scheme for mitigating ICI

The structure of the novel is shown in Figure 1.1 above. Here, the novel STTC-WH-STCC-OFDM system is introduced by combining the STTC-WH-ST-OFDM with the STCC-OFDM program. At the end of the transfer, the multiplexing (MUX) circuit is added and at the end of the receiver, a de-multiplexing circuit (DEMUX) is inserted. The maximum transfer value is provided among the lower carriers present in the OFDM block by pre-coder WHT. However, within two blocks, copies of the combined data are transferred externally with the help of a two-way transfer method. It is also possible to extend the MISO model to the MIMO structure here.

Vectors with coded symbols and their WH (d1 ', d2') and (-d1 '*, -d2'*) are transferred to two identical branches over time 1 and 2. IFFT is performed internally at the top of the branch and IFFT and conjugate ()$*$ operations are performed together at the lower branch. In order to retrieve the signal received from Tx1, FFT is employed by the upper branch at the receiver baseband [15]. However, conjugate operator is used first and then FFT is used to reduce the signal received from Tx2 at the branch below. To process the separation of the upper and lower branches, DEMUX is used here. For periods 1 and 2, with Tx1 and Tx2, the four available signal vectors are given below:

$y_{111}^{\prime}=F F T\left[h_{11} *\left(\operatorname{IFFT}\left(d_{1}^{\prime}\right)\right)\right]=H_{11} d_{1}^{\prime}$

$y_{121}^{\prime}=\operatorname{FFT}\left\{\left[h_{12} *\left(\operatorname{IFFT}\left(d_{2}^{\prime}\right)\right)^{*}\right]^{*}\right\}=H_{12} d_{2}^{\prime}$

$y_{112}^{\prime}=F F T\left[h_{11} *\left(\operatorname{IFFT}\left(-d_{2}^{\prime *}\right)\right)\right]=-H_{11} d_{2}^{\prime *}$

$y_{122}^{\prime}=\operatorname{FFT}\left\{\left[h_{12} *\left(\operatorname{IFFT}\left(d_{1}^{\prime *}\right)\right)^{*}\right]^{*}\right\}=H_{12} d_{1}^{\prime *}$

Further, hard decision variables are achieved through the assumption that across two consecutive time slots, fading is constant and these variables are shown below as:

$\underline{d_{1}}=\Psi^{-1} H_{11}^{*} y_{111}^{\prime}+H_{12}^{*} y_{122}^{\prime *}=\Psi^{-1} \Psi\left(\left|H_{11}\right|^{2}+\left|H_{12}\right|^{2}\right) d_{1}$

$\underline{d_{2}}=-\Psi^{-1} H_{11} y_{112}^{\prime *}+H_{12} y_{121}^{\prime}=\Psi^{-1} \Psi\left(\left|H_{11}\right|^{2}+\left|H_{12}\right|^{2}\right) d_{2}$

Here, the hard detected signal vector is represented by $d_{j}, j=1,2$. Through the receiver antenna Rx 1 this signal is obtained and then through Tx antenna $j$ it is transmitted. With the help of $\mathrm{CC}$, the channel impact to subcarriers is 
compensated after the IWHT and coherent combiner and detector. For novel STTC-WHSTCC-OFDM system, the new deconder algorithms are generated through equations (5) and (6). Thus, by concerning on each subcarrier, the hard-detected signal vectors enter the ML decoding algorithm.

$\underline{\hat{b}}=\arg _{Q} \min \left(\sum_{k=0}^{N-1} \quad\left|\underline{d}_{1}^{k}-Q_{11 k}\right|^{2}+\sum_{k=0}^{N-1} \quad\left|\underline{d}_{2}^{k}-Q_{12 k}\right|^{2}\right)$

Here, the $k$ th element of hard detection vector is denoted by $\underline{d}_{1}^{k}$. With respect to the receiver antenna $\mathrm{Rx} 1$ and the transmit antenna Tx " $j$ ", this hard detection vector is represented as $d_{j}, j=1,2$. Using two decision vectors which are $\underline{d_{1}}$ and $\underline{d_{2}}$ and including all possible code words that are $Q_{11 k}$ and $Q_{12 k}$ respectively, the two squared Euclidean distances are calculated separately [15]. Equation (7) shows the computation of final soft detected data bit vector that is denoted by $\underline{\hat{b}}$. The STTC, pre-coder WHT, and conjugate cancellation are combined with each other in this mechanism and it is seen that there is enhancement in the performance of BER in terms of MUX and DEMUX operations at $\mathrm{Tx}$ and $\mathrm{Rx}$, respectively, in comparison to other previously studied approaches.

\section{Experimental Results}

Filtering is a very common factor required within the radio communication systems as there is lot of noise present within them. For removing this noise from the electromagnetic signals, an effective filtering algorithm is applied.

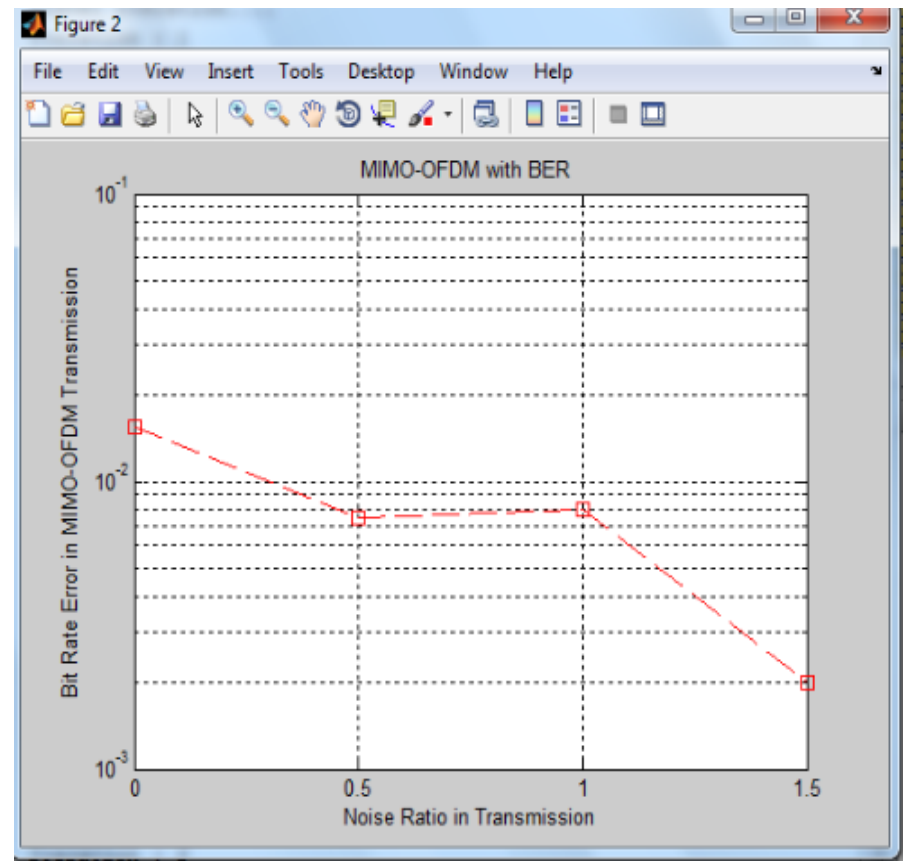

Fig. 2: Bit error rate due to noise

As shown in figure 2, the noise ratio within the MIMO-OFDM systems, the red line depicts the noise ratio. 


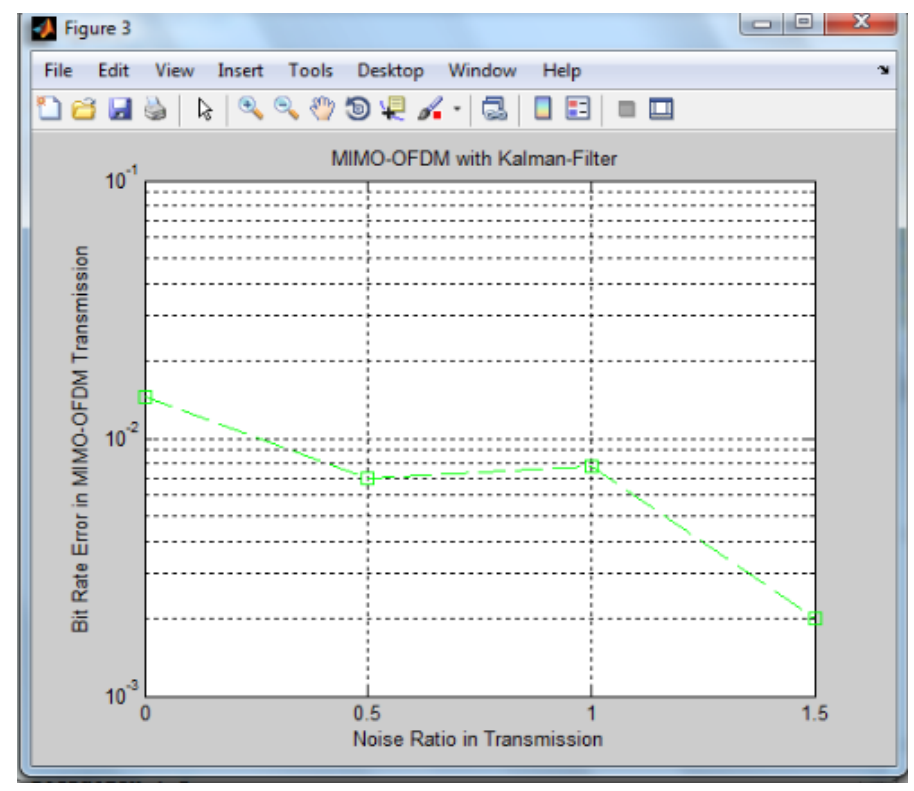

Fig. 3: Noise ratio with POLY-PHASE filter

As shown in figure 3, the noise ratio is represented with the help of Poly-phase filter within the MIMO-OFDM systems.

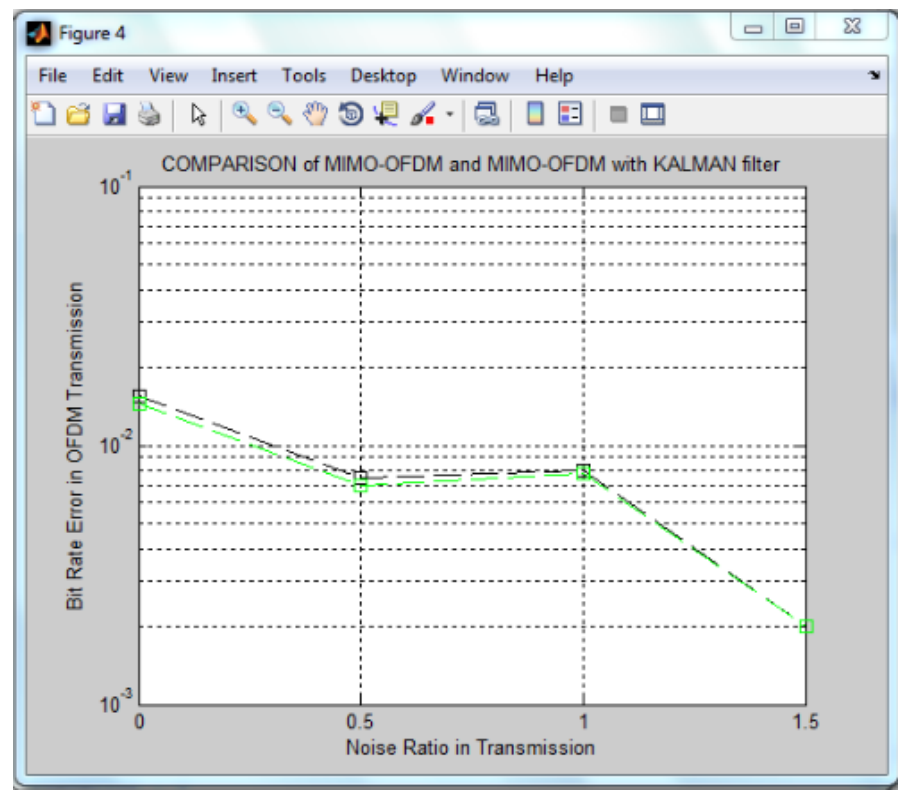

Fig. 4: Comparison between MIMO-OFDM and with POLY-PHASE filter

As shown in figure 4, the noise ratio is represented with black line and green line shows noise ratio with the utilization of POLY-PHASE Filter. There is reduction in noise ration and bit error with the utilization of Poly-phase filter in comparison to the genuine MIMO-ODFM systems. 


\section{Conclusion}

In this work, it is concluded A spectrally efficient digital modulation mechanism in which multiple carriers are present that are mutually orthogonal to each other over particular time is known as Orthogonal Frequency Division Multiplexing (OFDM) system. A sub-carrier is known as a carrier in which a pair of sine wave as well as cosine wave is involved. There are several closely spaced modulated carriers present within an OFDM signal. A propagation medium whose characterization is done through the wave phenomena is known as a mobile radio channel. The wireless fading channel has very high bit error rate. In this research work, the space-time trellis codes and poly phase filter is applied to reduce bit rate error. The simulation of proposed modal is implemented in MATLAB and results shows up to 20 percent improvement in the results.

\section{References}

[1]. F. P. Calmon and M. D. Yacoub, "MRCS-selecting maximal ratio combined signals: a practical hybrid diversity combining scheme," 2009, IEEE Trans. Wireless Commun., vol. 8, pp. 3425-3429.

[2]. Satoshi Gounai and Tomoaki Ohtsuki, "Performance Analysis of LDPC Code with Spatial Diversity," 2005, IEEE international conference on Vehicular Technology, pp 1-5.

[3]. Kwok Hung Li , Kwok Hung Li and Kah Chan The, "Performance Analysis of LDPC Codes with Maximum-Ratio Combining Cascaded with Selection Combining over Nakagami-Fading", 2011, IEEE, Transactions on Wireless Communications, vol. PP, no. 99, pp.1-9.

[4]. F. P. Calmon and M. D. Yacoub, "MRCS-selecting maximal ratio combined signals: a practical hybrid diversity combining scheme," 2009, IEEE Trans. Wireless Commun., vol. 8, pp. 3425-3429.

[5]. David Tse and Pramod Viswanath, "Fundamentals of Wireless Communication", 2005, Cambridge University Press.

[6]. Li Tang and Zhu Hongbo, "Analysis and Simulation of Nakagami Fading Channel with MATLAB", 2003, Asia-Pacific Conference on Environmental Electromagnetic, pp.490-494.

[7]. A. Tarighat and A. Sayed, "MIMO OFDM receivers for systems with IQ imbalances," 2005I, EEE Trans. Signal Process., vol. 53, no. 9, pp. 3583-3596.

[8]. Hen-Geul Yeh, Samet Yildı, "Space-Time Trellis Coded OFDM Systems in Frequency Selective Mobile Fading Channels", 2016, IEEE.

[9]. Neethu V, Ismayil Siyad C, "Performance Analysis of Diversity Techniques for OFDM system using Trellis Coded OAM-QAM union modulation", 2017 International Conference on Intelligent Computing and Control (I2C2).

[10].Houshou Chen, and Kuo-Chen Chung, "A Low Complexity PTS Technique Using Minimal Trellis in OFDM Systems", 2018, IEEE TRANSACTIONS ON VEHICULAR TECHNOLOGY, Vol. 67, No. 1.

[11].Samet Yildı, Hen-Geul Yeh, "The Performance Analysis of Space-Time Trellis Coded MIMO-OFDM Systems", 2016, IEEE.

[12].Ryota Yoshizawa and Hideki Ochiai, "Trellis-Assisted Constellation Subset Selection for PAPR Reduction of OFDM Signals", 2016, IEEE.

[13].Funmilayo B. Offiong, Sinan Sinanovi'c and Wasiu O. Popoola, "On PAPR Reduction in Pilot-Assisted Optical OFDM Communication Systems", 2016, IEEE. 